Página inicial: 157 - Página Final: 175

Tipo de artículo: Investigación.

\title{
Vicisitudes de los proyectos institucionales de atención psicosocial a víctimas del conflicto armado colombiano.
}

\section{Vicissitudes of the Psychosocial Attention Institutional Projects to Victims of the Colombian Armed Conflict.}

\author{
Por: Juan David Villa Gómez ${ }^{1}$ Laura Arroyave Pizarro², Yirley Montoya Betancur ${ }^{3}$, Alejandra \\ Muñoz ${ }^{4}$
}

Recibido: junio de 2016 Revisado: noviembre de 2016 Aceptado: diciembre de 2016

\section{Resumen.}

Comprender las experiencias subjetivas de los profesionales vinculados a proyectos de intervención con víctimas de violencia política en el ámbito psicosocial en la ciudad de Medellín y otros municipios del departamento de Antioquia, en relación con sus vivencias de malestar ético y desgaste emocional por su participación en estos proyectos, nos ha permitido identificar la forma como los proyectos están construidos, sus lógicas tecnocráticas y burocráticas, los problemas en los tiempos de ejecución y contratación, además de su escasa contextualización y sensibilidad a la realidad de las comunidades afectadas, realidad que termina convirtiéndose en generador de daño y revictimización, además de lesionar la integridad psíquica y ética de estos profesionales.

Palabras Clave. Víctimas, Intervención psicosocial, Reparación integral, psicología social, Acción con daño.

\begin{abstract}
.
Understanding the subjective experiences of the professionals connected with intervention projects with victims of political violence in the psychosocial environment in the city of Medellin and other municipalities of the State of Antioquia, in terms of their experiences of ethical discomfort and emotional drain, for their participation in these projects, has helped us to identify the manner how these projects are built, their technocratic and bureaucratic common-sense, the problems in the execution and hiring times, as well as their limited contextualization and sensitivity to the reality of the affected communities, such a reality becomes a creator of harm and revictimization, which also damages the psychic and ethical integrity of those professionals.
\end{abstract}

Key words. Victims, Psychosocial Intervention, Integral Reparation, Social Psychology, and Threatening Action.

\footnotetext{
1 Psicólogo, Magister y Doctor en Cooperación Internacional al Desarrollo. Docente / Investigador Universidad de San Buenaventura - Medellín. Grupo de Estudios clínicos y sociales en psicología, investigador principal. Contacto: juand.villa@usbmed.edu.co - judavigo@yahoo.com

2 Psicóloga Universidad San Buenaventura. Contacto: lau.arroyave@,outlook.com

3 Psicóloga Universidad San Buenaventura. Contacto: yirley.montoya@gmail.com

${ }^{4}$ Auxiliar de investigación, facultad de psicología Universidad San Buenaventura. Contacto: alejandramz.psic@,outlook.es
} 


\section{Introducción.}

Este artículo se enmarca en una investigación más amplia que tiene como propósito comprender las experiencias subjetivas de profesionales vinculados a proyectos de intervención psicosocial con víctimas de violencia política en la ciudad de Medellín y otros municipios del departamento de Antioquia, evidenciando vivencias de malestar ético y desgaste emocional por su participación en estos proyectos. En el proceso investigativo emergieron cuatro categorías: los aspectos vocacionales, las vicisitudes de estos proyectos, el desgaste emocional y el malestar ético de estos profesionales. El presente artículo se centra especialmente en las vicisitudes de los proyectos de intervención psicosocial con víctimas y otras poblaciones en condiciones de vulneración de derechos, según los relatos de los y las participantes.

Pablo Fernández Christlieb (2011) afirma que lo psicosocial es un significante que no logra recoger lo que con él se quiere expresar. No es ni una ciencia, ni un método, ni una forma de atención que trabaja sobre dos mitades, la mitad de lo psicológico (lo que corresponde a un individuo) y la mitad de lo social (lo que corresponde a muchos), ni tampoco el promedio entre lo uno y lo otro. Con este significante tampoco se termina haciendo referencia a la interacción entre un punto y otro, una cosa y otra, entre un individuo y otro, ni es un conjunto de actividades entre recreativas, colectivas, pedagógicas y comunitarias que se despliegan a lo largo y ancho de un territorio, en la mayoría de los casos sin norte, sin rumbo, sin marcos de comprensión; pero que se denominan psicosociales, porque logran reunir a un psicólogo, un trabajador social y un sociólogo para que hagan una tarea en algún lugar (Villa, 2012), con algún grupo de gente, de forma muy activa, pero sin tocar ciertos aspectos que corresponden a otro territorio, llámese éste: salud mental, clínica, terapia, porque eso ya no es psicosocial, sino todo lo contrario, es decir, lo individual. Es decir, lo psicosocial, sería lo grupal y lo comunitario. Pero acaso, ¿eso no era lo social?

Entonces, ¿Qué sería lo psicosocial? Fernández Christlieb (2011) afirma que es "la Situación", es decir, donde ya no hay individuos ni sociedad, es como el ambiente, pero eso es muy conductista, o el espíritu de una época o la mente colectiva, pero esto ya puede sonar a metafísica; o también, lo que está entre lo mecánico y lo simbólico, un emergente que aparece, que no se refiere ni al individuo ni a lo social, pero que se refiere a ambos, por eso "al intentar hablar de lo psicosocial, el lenguaje falla, como que no hay palabras con las cuales mencionarlo, porque ya todas están demasiado usadas" (p. 52). Al final nos quedamos con que es una forma de razonar, una forma de ver, una mirada, un modo de ser, que por lo menos intenta ir más allá de lo material, lo mecánico, lo lineal, lo causal y todo aquello en que nos metió la lógica moderna cartesiana y de la cual no acabamos de salir (cf. Villa, 2014b).

Así pues, no logramos decir lo que queremos decir cuando utilizamos el significante psicosocial, bien sea como adjetivo de atención, intervención o acompañamiento, o bien como sustantivo, referido a un marco epistémico y metodológico. En este texto el concepto psicosocial aparece referido a cuatro dimensiones: una ontológica, y desde allí hablamos de una perspectiva que guía la acción en una comprensión de lo humano como construido en escenarios relacionales, sociales, que conecta el denominado mundo externo con el interno y que reconoce las categorías de sujeto y subjetividad como centrales. En términos epistemológicos hablamos de enfoque psicosocial, lo que implica un acercamiento a los problemas de estudio con una 
inclusión del "observador" dentro de los escenarios que estudia y una mirada que enfoca la construcción de cada situación en sus marcos contextuales y socio-históricos (Cfr. Villa, 2012).

A nivel metodológico: lo psicosocial es una forma de estar con los sujetos que implica un acompañamiento procesual y no sólo una intervención de agentes externos sobre problemáticas que se abordan, esto conduce a una forma de la acción, en la cual los "expertos" en términos disciplinares y profesionales, se relacionan con el saber propio de personas y comunidades en sus propios contextos, generando un diálogo de saberes que posibilita conocimiento y acciones participativas, incluyentes y transformadoras. El cuarto nivel, el ético-político, plantea que hay una clara opción de transformación de situaciones de exclusión, opresión y violencia que generan sufrimiento a la población. En este sentido, las intervenciones no son neutrales, hay una implicación clara del agente profesional en procesos y dinámicas de las comunidades, de tal manera que su acción se enfoca a transformar las condiciones que vulneren su dignidad, calidad de vida y bienestar (Cfr. Villa, 2012; Blanco, 2012; Blanco \& Gaborit, 2014).

Estamos en un momento, particularmente en Colombia, donde "Casi que no se concibe proyecto de intervención social o comunitaria que no incluya en alguna de sus secciones, líneas, fases o procesos la palabra psicosocial como parte de la justificación, los objetivos, los resultados o las actividades" (Villa, 2012, p. 351). Por eso, se hace necesario revisar los proyectos de atención o intervención psicosocial y sus vicisitudes. Puesto que el uso desmedido del término desconoce buena parte de la tradición de psicología social crítica latinoamericana, donde se desarrolla como marco de acción (Una mirada que incluye el pensamiento de autores como Paulo Freire, Ignacio Martín-Baró, Maritza Montero, Marisela Montenegro, Fernando González-Rey, entre otros.); y la praxis misma de los proyectos presenta varias dicotomías y paradojas que van en contravía del marco anteriormente enunciado.

La primera paradoja o contradicción se da cuando se discuten los conceptos de atención y acompañamiento. El primero hace referencia a un marco de relación en la que hay un experto con el supuesto saber, y un "usuario/beneficiario" de un servicio de atención, en la cual hay implicada, normalmente, una relación vertical, de poder/saber, donde hay alguien que conoce las técnicas y metodologías y las aplica, desde afuera. Sin embargo, en el contexto de la psicología social crítica, hacemos referencia a un modo de la acción que implica acompañar, estar ahí, en la situación, que implica una construcción de vínculo horizontal, que empodera a los sujetos y genera transformaciones reales en sus vidas y en sus contextos (Cfr. Villa, 2013; 2014b).

A esta paradoja subyace otra, en relación con el modelo que guía la acción: la mirada centrada en la "atención" deviene del modelo biomédico, donde se desarrolla un proceso lineal en el que determinado un diagnóstico y aplicadas unas técnicas de tratamiento, sobrevendría la curación. De allí la necesidad de identificar, listar y clasificar los "males", trastornos o problemas que deberían ser "curados". De nuevo, se implica una relación vertical con un sujeto de supuesto saber, experto que dictamina el proceso. Mientras desde una perspectiva y enfoque psicosocial, se desarrolla una mirada que permite comprender que las situaciones de exclusión, violencia y sufrimiento, para ser transformadas, deben ser abordadas en su complejidad, incluyendo a miembros de la comunidad como participantes; tanto para identificar problemáticas y necesidades, como en la búsqueda de soluciones y alternativas que mejoren sus condiciones actuales. Todo esto implica miradas inter y transdisciplinares que permitan una acción integral. 
La gran paradoja estriba en que la mayoría de proyectos se plantean desde una lógica de racionalidad instrumental, técnica, donde los sujetos son individuos, guiada por principios de eficiencia y eficacia financiera, en un marco jurídico estrecho que define como contrato lo que es un proceso complejo. Y esto entra en contradicción con una lógica que admite el caos, la incertidumbre, el devenir y la complejidad de los procesos sociales, con sus ires y venires, con sus dificultades; entendiendo que los procesos son lentos y que implican paciencia histórica para poder actuar de forma transformadora (Blanco \& Gaborit, 2014; Villa, 2014b).

Es en este marco de paradojas y contradicciones donde los y las profesionales participantes en esta investigación descubren vicisitudes, dificultades y aporías de los proyectos de intervención psicosocial desde el Estado; por esta razón, recopilamos aquellas tensiones identificadas, gracias a su experiencia laboral, buscando relaciones de sentido y significado entre la forma como los proyectos están construidos y se desarrollan desde la planeación y ejecución hasta su evaluación, puesto que se suele evidenciar una distancia enorme entre lo que los proyectos y programas plantean y la realidad de la población beneficiaria y su contexto. Lo cual, en muchos casos, genera conflictos y dificultades en la ejecución de los profesionales, quienes terminan confrontados con dilemas éticos que no alcanzan a resolver de forma asertiva, con lo que deviene el desgaste emocional, y en muchos casos el distanciamiento de la tarea.

\section{Metodología.}

La investigación está enmarcada dentro del método cualitativo, cuyo carácter descriptivo y emergente, permite recoger sentidos y significados en torno a un problema planteado. Desde una mirada transdisciplinar nos acercamos a la complejidad del problema, trabajando en una perspectiva holística y con un enfoque fenomenológico-hermenéutico; que tal como lo explica Sandoval (1996) es una forma de investigación que se focaliza en la experiencia vivida, lo cual nos permite comprender y describir la experiencia en cada uno de los sujetos participantes.

En este ejercicio se llevaron a cabo 32 entrevistas semi-estructuradas, en profundidad, a profesionales de Medellín, otros municipios de Antioquia y Bogotá que trabajan en proyectos de "intervención" psicosocial con víctimas de violencia política en el departamento de Antioquia, en el marco de los procesos de reparación integral ofertados por el Estado según lo decreta la Ley 1448 de 2011. Se realizó un muestreo no probabilístico, tipológico e intencional con metodología de bola de nieve, que implica que un participante va remitiendo a otros y otras. Los criterios de selección fueron: profesionales que manifestaran desgaste en su labor, que expresaran tener vocación hacia el trabajo con población vulnerable.

Los relatos fueron analizados por el método de análisis del discurso, a través de categorías y matrices, que procede de manera analítica, avanzando en la interpretación de forma interactiva entre procesos inductivos y deductivos hasta llegar a la construcción de interpretaciones que posibilitan la construcción de los resultados de la investigación (Villa, 2014a). Los pasos son los siguientes: en primer lugar, un análisis de coherencia (intratextual), donde cada transcripción se analizó independientemente de las otras, lo que dio paso a la construcción de categorías. Posteriormente se realizó un análisis intertextual, tomando los contenidos que aparecían de manera reiterativa. Finalmente se realizó una codificación teórica que permitió organizar la información según un procedimiento axial (Flick, 2004). Luego de esto se desarrolló la teorización, con el material codificado y disponible, que enlaza la voz del investigador, a través 
de interpretaciones, hipótesis, desarrollos conceptuales, con los relatos de los y las participantes, constituyendo un texto que se teje en una unidad de sentido.

\section{Resultados.}

El análisis emergente tiene que ver con las condiciones institucionales que bloquean la intervención y generan frustración en el marco de los proyectos. Encontramos algunas significaciones comunes y relatos reiterativos en relación con las limitaciones estructurales en la construcción de los programas y propuestas institucionales. Según los y las participantes, este tipo de proyectos pareciera "cortado por la misma tijera" y se evidencian claramente: deficiencias en el acercamiento a los contextos, lo que implica, en muchos casos, que los profesionales incursionan en contextos y territorios que no conocen y con los cuales no se han familiarizado. Además de lo anterior existen limitaciones en la selección de personal, algunos profesionales no tienen habilidades para este trabajo y vienen como cuota política, manteniendo la lógica clientelar que suele acompañar las contrataciones en el Estado colombiano. Además de lo anterior se presentan problemáticas en los procesos de contratación y ejecución, limitaciones temporales que entorpecen el desarrollo de procesos; y una lógica asistencial que no posibilita acciones reparadoras ni transformadoras. Todo esto será desarrollado a continuación.

\section{Deficiencias en el acercamiento a los contextos.}

Fue recurrente que los participantes mencionaran ciertas deficiencias relativas al desconocimiento del contexto que las instituciones, especialmente las estatales, tienen al momento de plantear una intervención; posiblemente quienes planean las estrategias interventivas, lo hacen desde un escritorio y quizá pocas veces se han parado en el contexto o tienen la experiencia para hacerlo. Normalmente son "técnicos" en todo el sentido de la palabra, que tienen competencias en procesos de planeación, elaboración, ejecución y evaluación de proyectos, como campo temático y de aprendizaje, pero muy poco conocimiento de las realidades para los cuales éstos se diseñan (Cfr. Martín Beristain \& Doná, 1997).

De allí, se baja la propuesta a profesionales contratados para ejecutarla, quienes con frecuencia tampoco conocen a fondo el contexto en que desarrollarán su labor. En sus relatos, se expresaba con gran preocupación, puesto que este vacío venía incluso desde su misma formación universitaria, afirmando que las universidades no están preparando o formando profesionales que piensen en la realidad actual, sino que muchas veces forman a las personas desde un saber teórico, abstracto; en el conocimiento de unas técnicas, casi siempre instrumentales, lo que termina alejando al estudiante de su cotidianidad y de las posibilidades de reflexionar sobre su propia realidad (Cfr. Nussbaum, 2010; Villa, Arroyave, Montoya \& Muñoz, 2015)

¿Por qué pasa eso? Porque con todo respeto, el ambiente educativo escinde la realidad, y al aprender la disciplina y las técnicas siempre nos someten a una torre de marfil: yo pienso aquí, lo que quiero hacer allá; luego voy y lo aplico allá, y eso es un error. La universidad tiende a estandarizar el conocimiento. Lo que genera dificultades de algunos profesionales para la adaptación. (E20)

Hablar de contexto va más allá de estadísticas sobre orden público, número de muertes, secuestros, extorsiones, etc. Hablar de contexto es conocer las dinámicas que tiene una comunidad, en relación con eso que está sucediendo en su cotidianidad, conocer los signos, las formas de comunicación, las normas explícitas e implícitas, la forma en que se relacionan y los 
recursos que necesitan en relación con el macro-contexto, los juegos de poder a nivel local y sus vínculos con marcos sociales de poder más amplios (Martín Beristain, 1999). Conocer cómo la guerra ha alterado las relaciones, tanto interpersonales como intergrupales, la convivencia en ese lugar y la forma en que esto ha afectado la salud mental de la población (Martín Baró, 1989): "...porque pueden ser proyectos bien pensados para algunos territorios, pero los territorios, la gente y las dinámicas cambian; en este sentido, yo pienso que los proyectos no tienen la astucia de moverse para responder expectativas de la gente, no le sirven" (E19).

Marisela Montenegro (2001) menciona 4 preguntas que se deben tener en cuenta cuando se va a iniciar cualquier acción con una comunidad o un territorio: (1) ¿Cómo se construyen los problemas sociales? (2) ¿Cómo se construyen las soluciones? (3) ¿Cuáles son los entes relevantes para la solución? y (4) ¿Cuál es el cambio social posible y deseable? Sin embargo, al responder estas preguntas desde el marco de las denominadas estrategias y proyectos psicosociales, en los cuales han participado los profesionales entrevistados, podemos encontrar que no se plantean desde este lugar de construcción. Algunos intervienen desde aspectos que se han definido como problemáticos desde centros de decisión políticos y técnicos; no se privilegia el diálogo entre quienes diseñan las intervenciones, quienes actúan en campo y las personas afectadas, ignorando los contextos:

(...) cuando se tienen profesionales que no logran entender lo que ha pasado es difícil, no hay como esa sensibilidad sino simplemente cumplir un requerimiento legal y técnico... no se miran las particularidades de esa población, porque su historia no empezó cuando el hecho victimizante ocurrió, su historia comenzó mucho antes; entonces, si las entidades públicas no entienden esa historia, también se va quedar solo en asistencia (E24).

De esta forma, si los proyectos psicosociales no se plantean desde este diálogo, el papel que desempeñan los funcionarios, profesionales y facilitadores será poco apropiado, en la medida que son poco realistas y podrían llevar a realizar acciones con daño, porque "cuando una intervención de cualquier tipo ingresa en un contexto, ella se convierte en parte de ese contexto" (AVRE, 2010). Nos encontramos entonces con grupos de profesionales, que en muchos casos no tienen claro el contexto y en otros con procesos de análisis que no logran reconocer la realidad local y concreta. Por esta razón, cuestionan constantemente su quehacer, puesto que los procesos que se tienen planeados pareciera que no son efectivos, que se lanzaran al vacío y su impacto se ve seriamente disminuido en un contexto donde, además, persiste la amenaza, puede conducir a que se sienta que las acciones se hacen en vano; porque de cierta forma, se le imponen a la población "desde un lugar central, desde un escritorio, donde se ha definido lo que ellos necesitan" (E11). Esto pone en riesgo esa misma acción, evidenciando un problema que toca con la estructura del Estado, y que pareciera no tener pronta solución:

(...) pensar que uno puede rehabilitar mentalmente a una población, una comunidad en un contexto en donde aún hay violencia es inútil (E4). Todo el tiempo lo entorpece el hecho de que haya presencia de estos grupos, que tengan incluso más poder que el Estado, se convierte en un riesgo para las poblaciones, entonces si el grupo armado no legitima esas acciones, no legitima la participación de las personas en esto, eso va entorpecer el proceso, porque las personas no se van a presentar (E26).

De allí que se pregunten si se termina instrumentalizando a las personas víctimas para mostrar que se está haciendo algo, se está prestando la atención o ejecutando una reparación, pero al final, no deja de verse la contradicción implicada en estos procesos, especialmente en el marco de la ley 1448; puesto que, además, "en un contexto de alta violencia de grupos armados, en una 
situación de desigualdad económica y social tan fuerte como la de Colombia, garantizar que no haya repetición es muy difícil” (E20).

En suma, el primer déficit de los proyectos psicosociales en los que han participado los profesionales entrevistados es que son pensados, proyectados, seguidos y evaluados desde el escritorio. Por eso plantean la necesidad de construirlos y ejecutarlos desde el contexto y con las personas, quienes deben ser partícipes de sus propios procesos de desarrollo y empoderamiento.

\section{Delimitación temporal y sus efectos en los acompañamientos psicosociales.}

En relación con lo anterior, también se encuentra, entre estas condiciones, una problemática en relación con los tiempos: de planeación, ejecución, contratación del proyecto y de los profesionales como elemento nuclear de la intervención psicosocial: "La reparación transformadora está pensada desde transformar tejido social y esto supone, afrontar el dolor; pero para eso, paradójicamente, en los proyectos no hay los tiempos adecuados" (E17). Al parecer hay dos configuraciones: unos son los tiempos de la gente, los de la comunidad, incluso los tiempos del daño padecido. Otros son los tiempos de una lógica instrumental que está implicada en la mirada metodológica de planeación y ejecución de proyectos, en la cual se traza un objetivo para dirigirse al mismo, similar a como sucede en un proceso tayloriano de producción, organización de tiempos y movimientos (cf. Bello \& Millán, 2005).

Esta lógica es válida en ciertos contextos de tipo productivo y financiero. Pero cuando la aplicamos a los procesos humanos, pensamos que, por ejemplo, hacer intervención psicosocial, construir comunidad, construir paz, es como si fuera producir un bien de consumo en el mercado. Así, todo el personal termina corriendo para cumplirle a un indicador, a un resultado; lo que puede generar daño, porque se tiene que demostrar que "se atendieron" a X número de personas en una cantidad de tiempo, en muchos casos: "llevándose por delante a todo el mundo" (E29).

La situación es más o menos esta: se detecta un problema, se trazan unos objetivos, que se abordan con una metodología (llámese marco lógico, planeación estratégica u otra), se definen actividades y se supone que por estas acciones tiene que haber unos resultados en un determinado tiempo, seis meses o un año (En los tiempos que corren un proyecto de más de un año es además de una excepción, casi una bendición). Ahora bien, este proyecto necesita financiación, y quien financia, llámese cooperación internacional o Estado, quiere darle el mayor rendimiento a los mismos. Por lo tanto, ubica metas e indicadores que justifiquen la inversión. En términos del modelo occidental de construcción del mundo esto parece lógico, casi que justo:

(...) se plantean unos objetivos anuales por parte del Estado y eso está bien. La cuestión en realidad es que se contrata al personal para lograr esos objetivos en pequeños períodos, entonces digamos hay que intervenir en el departamento de Antioquia 7.000 personas o 7.000 familias y para eso se contrata tanto personal durante tres meses, a mí me dieron 3 meses para atender 250 personas aproximadamente de 4 o 5 sesiones, eso es una cosa casi imposible a menos que tu inviertas todo el tiempo que tienes durante esos tres meses... Si hubiese más tiempo, para los objetivos, uno lo podría hacer con mejor calidad, cumpliendo con todo, con ciertas medidas de protección o autoprotección para uno a nivel mental. Y la cuestión es qué es una visión puramente económica (E29). 
De allí que puede generarse en los y las profesionales un sentimiento de incompletud, que se asocia a una sensación de fugacidad en las intervenciones que terminan siendo vistas, por ellos mismos, como acciones superficiales; puesto que evidencian que en muchas ocasiones el dolor, el sufrimiento y las realidades desestructurantes de los sujetos y las comunidades, siguen sin transformación, "lo que yo siento es que cuando uno trabaja con víctimas o con el sufrimiento del otro, pienso que esa experiencia necesita vivirse, pero también reflexionarse, pero también incorporarse, (...) entonces yo siento que es muy poco el tiempo para esto" (E5).

La mirada institucionalizada se centra en definir claramente el proyecto, los procedimientos, las técnicas, estandarizar las formas de acción y formatear la información para poder tener mayor control y predicción sobre lo que se hace, los tiempos de ejecución y los tiempos para consignar la información. Así pues, se contratan profesionales para que desarrollen la tarea. Estos profesionales solicitan técnicas y procedimientos para poder hacerlo, se entrenan en esas formas, y empiezan a ejecutar. Solamente que en medio no está en juego la producción de galletas, jabones o tornillos, sino el sufrimiento concreto de personas y comunidades.

Primero hay que reestructurar la forma cómo están diseñadas las instancias de atención, protección y garantía de derechos; porque hay un montón de proyectos que no responden a las necesidades de la gente, responden más a necesidades políticas, cumplimiento de programas, leyes y normas, pero, realmente son un cumplimiento sordo y ciego (E4).

Esa lógica instrumental del tiempo rompe a los miembros de las comunidades, rompe a los profesionales que actúan en estos contextos con estos dispositivos. Va en contradicción con la mirada diferencial y el enfoque de acción sin daño que caminan de la mano con lo psicosocial. Como se logra ver, cuando se aplica esa lógica a los procesos humanos, la lógica de los formatos, de realizar todo en el menor tiempo posible, pasando incluso por encima de las personas, olvidando la importancia de construir comunidad, construir resistencia, y de construir la paz; es una situación dolorosa y crítica para los profesionales entrevistados.

(...) todos los días pensando en el número, miro como hago para cumplir, pero que yo esté sentada con alguien y pensando este es el número 84 y me faltan 522, eso nos va a hacer contar la gente como contando reses, y ese no es el sentido. Siempre vamos a estar detrás del requerimiento numérico y se nos va a olvidar qué es lo realmente importante (E12).

Así pues, contextualizarse, generar empatía, tejer confianza y llevar procesos, son cosas que requieren tiempo. Es más, si contrastamos, veríamos que los hechos victimizantes que han roto lazos sociales y han generado trauma en este país, no son de días ni de meses, por lo que no se puede esperar realizar acciones reparadoras en un lapso de tiempo tan reducido, se necesita tiempo para estar y acompañar y ahí es donde se reconstruye. Por su parte, la lógica del indicador, del resultado a corto plazo, rompe los procesos con la gente (Villa, 2014b): "una cosa en tan corto tiempo es muy difícil que sea reparadora. Hay cosas que sí se logran, pero hay tanto sufrimiento, que es muy difícil pensar que en 4 o 5 meses se logre hacer algo" (E5).

Los profesionales entrevistados afirman que han hecho el esfuerzo por acompasar los tiempos entre lo que demanda la institución y la gente; para ellos y ellas es algo que "depende de ti como profesional; porque la institucionalidad demanda otras cosas" (E13). Sin embargo, esto genera una sobrecarga que, en muchos casos, va más allá de sus posibilidades de actuación y los pone en una situación, donde las instituciones estatales son ciegas y sordas a los contextos, 
necesidades y procesos de las comunidades; que, insatisfechas, con la premura de proyectos enmarcados en una lógica instrumental, exigen el respeto a su dignidad y a sus derechos, lo que al final genera desgaste emocional y malestar ético.

Así pues, la realidad continuamente señala la paradoja de un profesional que encarna la institucionalidad y al mismo tiempo evidencia sus sombras: éste discrepa con su rol asignado y tiene un lío ético en el que, o "agrede" a la población con lo que el proyecto le dice que debe hacer, imponiendo tiempos y movimientos para lograr metas y resultados; o se agrede a sí mismo, imponiéndose tiempos excesivos de trabajo que le permitan acompasar esta profunda contradicción, puesto que es él o ella quienes ponen la cara delante de la comunidad y se convierten en el rostro de la institución, recibiendo el malestar y el desacuerdo ante las inconsistencias del proyecto:

Eso quema... es muy paradójico porque soy institución y es como si estuviera atacándome, pero, ¿Cómo te atacas a ti misma? Diciendo que la institucionalidad es una mierda y que no sirve para nada porque deja a las pobres víctimas tiradas, y yo soy institución, eso también quema (E16).

Es importante añadir un nuevo problema: la permanencia de los profesionales dentro de los proyectos, la continuidad de la acción y de las personas que la ejecutan, no hay una mirada de largo plazo: como los proyectos desarrollados por el Estado no tienen continuidad en sus tiempos de ejecución, en relación con las vigencias presupuestales y la inmensa cantidad de controles establecidos, con la consiguiente interrupción de la intervención, esto trae como consecuencia, cambios de personal, pérdida del capital humano que, como se decía anteriormente, se contextualiza en medio de su praxis, teniendo que llegar nuevos profesionales a "reinventar" lo ya realizado y volver a comenzar en un reproceso de nunca acabar:

(...) hay muchísimos errores, a veces la discontinuidad de procesos hace que vuelvan y empiecen reprocesos, ensayo y error, ciertos procesos, se paran, vuelven y empiezan... (E28). El tema de la contratación es fatal, por ejemplo, se acabó PAPSIVI en noviembre de 2015, pasó diciembre, enero, febrero y marzo, van cuatro meses, es probable, que ahora ya se haga la asignación de recursos, se va todo abril, y probablemente en mayo empiece de nuevo, entonces entra en eso: una inestabilidad laboral que a la gente no le motiva quedarse, y cuando se queda es pura incertidumbre (E30).

Recapitulando, una de las condiciones mínimas para la ejecución de un proyecto es el tiempo: abrir procesos que respeten el ritmo de la gente, podría generar las posibilidades reales para la transformación a la que se aspira desde la institucionalidad. De acuerdo con los y las participantes en esta investigación, hasta que no se genere un cambio en esta perspectiva, en aras de la ética y la responsabilidad, habrá que ser muy claro con la población, para que no se generen falsas expectativas ante marcos temporales que limitan la acción y transformación psicosocial: “(...) la reparación transformadora está pensada desde transformar tejido social y el dolor, pero para eso no hay los tiempos adecuados... definitivamente no se da el tiempo para acompañar a la gente y para generar unas acciones transformadoras" (E17).

\section{“La lógica del formato": evidenciar lo que no se alcanza a hacer.}

La lógica instrumental se sigue profundizando: no solamente hay un problema en la construcción del proyecto desde el escritorio y sin contexto, ni solamente una discordancia profunda en relación con los tiempos; sino que esto se complementa con la llamada lógica del formato. Desde su marco positivista y tecnocrático, esta lógica exige que puedan evidenciarse 
todas las acciones que se ejecutan. Para ello se van creando múltiples formas de registro, de tal manera, que cualquier actividad o proceso sea susceptible de ser verificado, controlado y constatado. Así pues, los profesionales deben invertir buena parte de su tiempo en llenar formatos, que plasman información sobre los beneficiarios del proyecto y las acciones del mismo. Esto genera una pregunta significativa: cuando el tiempo es breve, la cantidad de personas es masiva, la magnitud del daño supera cualquier cálculo, ¿qué significa pretender registrar cada uno de los movimientos y acciones? Es más, ¿qué significa llevar a cabo un proceso de intervención psicosocial donde llenar un formato puede implicar igual o más tiempo que atender y acompañar a las personas, familias y/o comunidades?

Los profesionales entrevistados afirman por unanimidad que los formatos, además de ser muchos, terminan siendo un obstáculo para la misma intervención psicosocial que se quiere desarrollar. Además, están los informes, encuestas, entrevistas, caracterizaciones, etc. que hacen parte de su labor, y que muchas veces, para las instituciones y los entes que las fiscalizan, se convierten en la piedra angular de las atenciones:

¡La caracterización tiene 10 hojas! A mí me parece una exageración, honestamente... entiendo por qué está, por qué funciona de esa manera, no estoy diciendo que no sea importante, porque de eso tiene que quedar un registro (...). Pero, lo que pasa es que es el formato, del formato, del formato... y es para hacerlo a cada persona, a cada comunidad, a cada familia, hay que llenar un formato distinto, entonces es una cosa que te quita tiempo, yo he trabajado desde lo público; y en lo público, lo que cuenta es el formato, es la evidencia, de que vos hiciste tu trabajo, tan sencillo como eso (E14).

La técnica y una lógica positivista y legalista de la evidencia va cobrando autonomía frente a los actores que la crearon, lo que era medio se convierte, en fin, como un Frankestein todopoderoso frente al cual los profesionales terminan rendidos, porque es lo no negociable, lo evidenciable, lo medible, lo cuantificable y lo verificable por entidades de control; así, toma vida y pasa a ser el amo. En una nueva paradoja se termina trabajando para esta evidencia, para el formato, más que para servir y apoyar a las personas y comunidades. Lo que trae consigo una anulación del profesional, en detrimento del objetivo y el sentido al que sirve, cayendo, en algunos casos en el sinsentido de la propia labor, porque, al final, lo único que cuenta es "la evidencia", sobre lo que se valora y evalúa su labor.

$Y$ en ese sentido terminamos haciéndole juego a no reconocernos, a no reconocer la gente con la que trabajamos, a no reconocernos entre nosotros como profesionales, entonces también vamos cayendo en el juego de invisibilizarnos, en el juego de llenar fichas, de hacer papeles, de hacer las cosas a la carrera, muchas veces sin una alcanzar a sentirse, a conectarse con el otro, entonces a eso yo lo llamo, ser un idiota útil (E19).

El problema no es tanto llenar el formato, ni tampoco los informes mensuales; sino que se convierte en un fin, sobre el cual empieza a girar toda la acción. Lo más grave es que, a pesar de tanto registro y evidencia, la experiencia de vida de la gente y la experiencia del encuentro con el profesional se invisibiliza, queda por fuera, no logra registrarse ni circunscribirse a esa lógica; dejando por fuera lo fundamental de una acción psicosocial con sentido: la relación, la construcción del vínculo y el proceso mismo de acompañamiento y transformación. Por esta razón los y las profesionales participantes mencionan que esta lógica, además de ser un factor que cansa y agota físicamente, en ocasiones, incluso, agrede a las personas al momento de hacer el registro: "para trabajar con la gente, la postura debe ser serena y ponerse en los zapatos de esa 
persona, no atacarlos. No... es que tienes que dibujar, necesito un reporte. No, la persona tiene su tiempo y su espacio" (E15).

Debido a esta situación, se preguntan lo siguiente: si el Estado, quien es el principal responsable de la reparación, se encuentra plagado de exigencias de este tipo para poder mostrar cifras esperanzadoras en apariencia, ¿al servicio de qué está mi trabajo? ¿Qué es lo importante aquí? ¿A qué le estoy haciendo el juego?

(...) que nos digan: pongan en una lista todas las personas que tienen. Se envía ese reporte a Bogotá. Entonces esa persona aparece atendida para el Ministerio de Salud... ipero no! esa persona no se atendió, hablamos de lo comunitario, pero no se atendió, pero ya está atendida para el Ministerio en una meta, ¿pero la persona qué? (E1).

Como consecuencia de esto, los profesionales se sienten sobrepasados, porque la misma estructura de atención no les permite cumplir su labor ni el fin para el cual fueron contratados; pues "este es un sistema que te saca, si no entras en la lógica de ellos, de funcionarios, técnicos, que sólo cuentan números y no atienden humanos, el sistema te saca" (E4). Algunas veces, piensan y sospechan sobre estos mismos programas y proyectos, porque pareciera que la apuesta política y estatal es por la cantidad, no por la calidad de la reparación, por cumplir con unas metas gubernamentales donde las cifras y la medición "estadística" es más importante; ofreciendo, en muchos casos, tiempos irracionales a los procesos psicosociales, lo cual va en contradicción con su convicción vocacional (Cfr. Villa, et. Al, 2015).

Se pone entonces, en tela de juicio la calidad de la atención, se pierde el propósito cuando la cantidad de personas atendidas es lo que más importa. Uno de los participantes menciona los efectos que esto puede tener en un profesional que teniendo clara su vocación, termina al servicio de algo totalmente diferente: "lo miden por el número de personas que atiende, sin importar la calidad, cómo se pone ahí como profesional, cómo fue su proceso, yo creo que termina secándose el alma a cualquier profesional que tenga un interés diferente al salarial" (E5). Por eso Bauman (1989) afirma que es importante rescatar que sólo los seres humanos "pueden ser objeto de enunciados éticos, pero una vez se les reduce a cifras, los seres humanos pierden esa capacidad" (p. 127), porque la administración burocrática reduce a las personas a meras cifras. Así pues, cuando se enfatiza en lo técnico y se pierde la relación, los profesionales empiezan a desgastarse:

(...) estos programas cosifican al otro, pero ya estamos cansados de eso, produce malestar cosificar al otro... y lo psicosocial es acompañamiento y tiempo con la persona. Nosotros tenemos unos deberes, pero produce resistencia ejecutarlos, es como si todas esas personas que apoyamos las transformamos en una cifra, una tabla de Excel (E7).

Por esta razón, se han visto en la situación de tenerse que "dividir" entre estas lógicas institucionales por un lado, respondiendo a formatos, cifras, metas; y por el otro, según el propósito del proyecto, trabajar en procesos con las comunidades y personas en espacios temporales muy breves, tratando de adecuar períodos de ejecución e indicadores, además de las órdenes de quienes dirigen los programas, que se mueven de forma vertical y autoritaria en el ejercicio del poder; lo que se debe acompasar, además, con demandas de líderes y representantes de las comunidades, o con pedidos que la gente hace: 
(...) yo creo que nosotros tenemos un discurso institucional muy esquizofrénico (dividido/escindido). Pero digamos que esto no es una malignidad "per se" de la institucionalidad, que quiere que vos te escindas, no. Sino, que vos, como intermediario del servicio, te sentís también como escindido por decirlo así (E20).

$\mathrm{Al}$ estar el profesional en ese "tira y hale", va a apareciendo un desgaste significativo, una experiencia que le absorbe tiempo y disposición, entonces, por responder en ambos escenarios, se agota física y mentalmente. Además de esto, cuando está implicado desde una convicción ética, se ve afectado, cuando se ve obligado a dar respuestas maquilladas para apaciguar la gente y quedar bien con la institución: “... a nosotros nos meten un cuento de simplemente haga, llame, contacte, hable, entregue y ya chao; y vuelva con otra persona a hacer lo mismo, con otra familia, entonces muchas veces la lógica institucional no es la de las personas o del país" (E18).

\section{De la burocracia al clientelismo.}

Estos problemas tienen relación con una lógica que devela un orden social establecido y una racionalidad que resulta, además de deshumanizante, ineficaz e ineficiente, contrariando sus propios principios, para lograr los fines que se propone. Pero de acuerdo con los participantes, a esta problemática se le añade otra que tiene relación con prácticas politiqueras enquistadas en el funcionamiento del Estado. Es decir, además de dificultades de orden metodológico, epistemológico y práctico, las dificultades son mayores cuando se constata en terreno la incompetencia de algunos de los colegas que son contratados para este tipo de proyectos.

En primer lugar, aducen deficiencias en relación con lo que podría ser una selección de personal, puesto que, en el ejercicio de su quehacer, se han encontrado con la falta de preparación de algunos profesionales, personas que no están lo suficientemente preparados "y no son los más adecuados o idóneos" (E16) para esta labor. En un texto anterior (Cf. Villa et. al., 2015) se planteaba que un elemento clave para estos profesionales era vincularse a estos proyectos desde un horizonte de sentido, recogiendo elementos vocacionales como el deseo, el gusto por acompañar a otros, el interés por trabajar con la comunidad, con la gente. Desde este punto de vista, implicaría que no sentir este trabajo, no tener esta convicción y realizarlo como la consecución de un empleo en el marco del pago de favores políticos, hace que el perfil de estas personas, quizá podría no ser el adecuado para este campo de acción: "ese tipo de personas no se percatan, no es desconocimiento, es pura negligencia, puro automatismo, necesitan estar en ese cargo y ya, no importa nada más, trabajar con esos funcionarios es muy complicado" (E4).

La llamada "rosca" o clientelismo implica que la persona contratada no obtiene el cargo por sus competencias y experiencia, ni por sus habilidades y carisma para el trabajo con una población seriamente afectada en su integridad y dignidad. No importa quién trabaje, cómo trabaje, qué hace, solo importa que es el "amigo de...", el conocido o pariente de..., que se recomendó para trabajar, sin tener las competencias para el cargo:

A mí me genera mucho dolor que elijan mal los funcionarios, pienso que ese es el primer acto de corrupción, que gente que no cree en la reparación esté trabajando aquí... legitiman nuevas formas de violencia, solamente que están amparados por una institución, pero es la misma violencia: "me vale m..., a mí no me importa, pero las cifras van subiendo". Yo siento mucha desilusión, no he podido descifrar la palabra, creo que a veces trato de darme la explicación y de pensar que tal vez esa gente también ha sufrido, y por eso se comporta de esa manera o que tal vez el sistema los absorbió, se les 
robó la ética y se les comió su sentido de humanidad; y por eso actúan de esa manera. No sé ni siquiera cual es la emoción, sé que duele, creo que es eso.... (E22).

De acuerdo con esto, pareciera que para algunos profesionales entrevistados es absurdo y no tiene ninguna justificación el hecho que este tipo de situaciones sucedan en las instituciones y programas, puesto que son elementos que generan retrasos, entorpecimiento, bloqueos, y en muchos casos, malestar: “...es como estar en el país de las roscas, así funciona Colombia. Pero es como una doble falta porque el Estado crea un programa para atender bien a las personas y eso no se hace. Entonces es doblemente inepto" (E3).

Además de lo anterior, se pudo ver que dentro de las condiciones institucionales que dificultan la realización de procesos, el tema de las garantías laborales es generador de nuevas dificultades, puesto que no son las mejores: si bien para varios de ellos trabajar con la gente es una pasión, un sentido de vida y un compromiso que llevan dentro (Villa, et Al. 2015); esta labor se ve opacada, por condiciones laborales precarias. El modelo de contratación es uno de los principales problemas que se aducen en los relatos, tener contratos por unos meses y luego esperar un tiempo prolongado para volver a ser contratado. Vivir en inseguridad laboral constante, saber que valen muy poco los resultados logrados y mucho menos los procesos acompañados, puesto que siempre lo que define la acción será el monto presupuestal y los intereses desde los lugares centrales de poder. De tal manera que los y las profesionales terminan siendo "piezas" que se pueden cambiar dentro del engranaje de una máquina:

Es un montón de angustias por dentro, cómo no nos va a afectar el desempeño, si estás pensando: ya se me va acabar el trabajo, voy a estar cuatro meses sin trabajo, con lo que me pagan no me alcanza, es un asunto que es imposible no pensar que no va a afectar (E12). Entonces vuelve otra vez y entra en eso, es una inestabilidad laboral que hasta la gente no le motiva quedarse ahí y cuando se queda es como una incertidumbre (P-E30).

\section{Los problemas estructurales.}

Según lo visto, existen unas deficiencias estructurales del Estado para atender a la población. Los relatos evidencian la frustración y la impotencia por no poder contribuir a transformar situaciones estructurales y básicas de la población; puesto que las acciones y propuestas chocan con un muro enorme: la lógica instrumental no escucha y el sistema burocrático y clientelar es lento para darse cuenta y actuar de forma distinta. De tal manera que, poco a poco, sienten que la lucha que se emprende no llega al objetivo, siempre están recomenzando procesos, atendiendo urgencias, mientras la situación real de la gente se mantiene en el tiempo. Esto los lleva a sentirse desesperanzados, concluyendo que “...el Estado no funciona” (E21), y está lejos de cambiar porque su rigidez parece inexpugnable,

A veces pasa eso, frustraciones o momentos de derrota por condiciones institucionales. Yo creo que si no de manera intencionada, sí por omisión. Porque la institucionalidad es muy lenta para reaccionar, depende de planes anuales que son muy rígidos. Entonces esta cosa se hace y se hace así (E20).

Hechos como la falta de cobertura en servicios de salud, la pobreza extrema, las carencias nutricionales, las deficiencias educativas, la imposibilidad de un transporte digno, la falta de oportunidades, el desempleo, entre otras situaciones estructurales se convierten en limitantes, no 
sólo de la ejecución de los proyectos (porque las personas faltan constantemente a las actividades programadas), sino también porque no se sabe si estas acciones desarticuladas y descontextualizadas son pertinentes, pues pretenden la reparación y no toman en cuenta la experiencia de la gente, su contexto; de tal manera que todo este marco de pobreza estructural y de limitantes estructurales del Estado ponen en entredicho acciones que apuntan a otro tipo de dinámicas: "Las víctimas deben tener presupuesto, la salud es pésima. Hemos encontrado municipios donde las víctimas sólo tienen un día asignado para pedir la cita, o sea que ellos solo tienen un día para enfermarse" (E1).

En este aspecto hay que comprender también, cómo la presencia prolongada de profesionales e instituciones en las comunidades van generando cambios en su cotidianidad, se van volviendo parte integral de su vida, y muchas veces son identificados como aquellos que los ayudarán a acceder a otros sistemas, de los cuales quedan al margen; pero, van perdiendo credibilidad al ver que sus situaciones precarias no cambian, lo que a su vez genera en estos profesionales malestar en su fuero ético, manifestado en estar y sentirse "quemado", por estar representando una institucionalidad que no responde a estas dimensiones estructurales:

(...) la gente sabe cómo funciona y saben recuperarse, pero uno de los problemas básicos es que las necesidades básicas no las tienen, es muy teso ir hacer un taller con una comunidad que tiene hambre, los chiquitos sin haber almorzado, ahí es cuando uno se enfrenta a estas situaciones y uno, ¿cómo hace para hablarle de sentimientos y emociones cuando lo que esta señora tiene es hambre? (E7).

\section{Discusión.}

De acuerdo con los y las profesionales participantes en esta investigación se puede plantear que la elaboración de proyectos de intervención y atención psicosocial a víctimas está padeciendo de varias problemáticas que pueden reconocerse dentro de los resultados que se han establecido con anterioridad. Si en realidad el Estado Colombiano quiere realizar acciones transformadoras de un orden social de violencia, y realizar una reparación integral que posibilite la dignificación de las víctimas, la recuperación emocional y la reconstrucción del tejido social, especialmente de cara al posconflicto armado debe transformar estas prácticas referenciadas.

Puede afirmarse que este tipo de proyectos son auto-referidos, sólo se miran a sí mismos y a los procedimientos metodológicos establecidos previamente, desconociendo, en buen medida, los contextos; en un proceso epistémico que recuerda al denominado "idealismo metodológico" que desarrolla un mimetismo "cientista" hacia metodologías de corte funcionalista y abstracto, además de una visión homeostática, a-histórica, de los fenómenos suponiendo que la "buena" realización metodológica implicará una acción que impacte positivamente en la realidad (MartínBaró, 1998, 2006). Según lo trabajado en esta investigación, muchas veces los profesionales son formados desde un saber teórico, abstracto y técnico, lo que termina alejándolo de su cotidianidad y de las posibilidades de reflexionar sobre su propia realidad (Cfr. Nussbaum, 2010; Villa, et Al., 2015).

En este sentido Nussbaum (2010) apunta que el problema puede ser sistémico, puesto que se están formando más profesionales para producir renta y favorecer una visión economicista del mundo, en una educación que parece necesitar más "personas que hablen una jerga grupal, que se comporten como burócratas dóciles y que también ven el mundo actual como tales (P. 46)", de tal manera que no se cultiva ni el juicio, ni la reflexión, ni la mirada crítica, ni la imaginación 
(Cfr. Bauman, 1989); con lo que, en muchos casos, quienes egresan de las universidades no están preparados para asumir retos tan fuertes y profundos como los que implica atender a víctimas del conflicto armado colombiano, con todas sus complejidades.

Desde una perspectiva psicosocial crítica más que intentar aplicar teorías, pretendidamente universales, y metodologías estandarizadas y homogenizadas a diversos contextos, los procesos de construcción del conocimiento y la guía para la intervención surgen mucho más desde la praxis, entendida ésta como un hacer consciente, como un ejercicio artesanal en el que se va conociendo la realidad por su estar en ella, actuar sobre ella y leerla desde dentro, movilizando acciones que posibiliten su transformación o cambio. Ahora bien, profesionales formados en teorías y métodos descontextualizados, siguiendo patrones ajenos a nuestra realidad, tendrán mayores dificultades para actuar e intervenir en los contextos complejos de la realidad colombiana (Martín-Baró, 1998, 2006; Blanco 2012; Blanco \& Gaborit, 2014).

Por eso, Montenegro (2001) propone que, desde las intervenciones participativas, el diálogo tanto en el conocimiento científico como en el conocimiento popular, son igualmente relevantes para generar transformaciones y procesos de empoderamiento colectivo que implican formas de afrontamiento, resiliencia y reconstrucción del tejido social ante las consecuencias del conflicto armado. En resonancia con Martín-Baró (1983) lo psicosocial es una bisagra entre lo social y lo individual, que atiende a un sujeto en contexto, es decir, no se concibe una acción psicosocial en un contexto sin sujetos o unos sujetos sin contexto (cf. Villa, 2012, 2014b).

De aquí se desprende un segundo problema: la planificación detallada del tiempo, a través de la planeación estratégica y de los cronogramas de trabajo, se contrapone a los tiempos de la gente, la magnitud del daño y la cantidad de personas, familias o comunidades que se deben atender. En esta situación paradójica, el tiempo escasea para que los profesionales puedan construir lo que Veena Das (2008) llama una "comunidad moral".

Restituir el habla a la sociedad (comunidad moral) es uno de los objetivos que nos debemos proponer cuando en las comunidades dolidas lo único que queda es el silencio. Este concepto que propone Veena Das, da cuenta de un espacio, más allá de lo terapéutico, donde las víctimas se conozcan, reconozcan su dolor, puedan escucharse, dialogar para reconstruir las historias personales a través de un ritual público de duelo. Cuando el sufrimiento, el dolor, la queja, se ponen en el escenario colectivo, se da la posibilidad de expresar la ira frente a la pérdida; en este sentido, el dolor se puede articular en lo público, expresando "me duele", ante esta comunidad moral. Aquí entonces radica su fuerza sanadora.

Y cada persona, cada comunidad es una historia, es un mundo de significación. Cada lugar es una idiosincrasia, una cultura, un tiempo de acercamiento. Son personas afectadas que se acostumbraron a callar, que tienen miedo, desconfían de todo y de todos porque esto les ha permitido sobrevivir (Villa, 2014a). Las personas y las comunidades son más que víctimas, son más que eventos violentos, son más que historias complejas, son resistencia, son dignidad; la gente no tiene por qué adaptarse a tiempos y procesos marcados desde la razón instrumental (Blanco \& Gaborit, 2014).

Sin embargo, se impone la lógica de la tecnocracia que atraviesa estos procesos humanos. Amalio Blanco (2012) plantea una contradicción entre esta lógica, propia de la razón instrumental, y la lógica de lo que denomina "razón compasiva": una óptica diferente cuando se 
trabaja en proyectos con la gente, que se traduce en una mirada emancipadora que posibilite transformaciones significativas. Y esto pasa por tener tiempo para el encuentro, para la escucha, para el acompañamiento, para estar. De acuerdo con Martin Beristain (1999, 2010); Clancy \& Hamber, 2008; Villa (2013, 2014b) y Bello \& Millán (2005), este tipo de acciones puede presentar un mejor pronóstico de transformación, tanto desde la perspectiva de los profesionales involucrados en el proceso, como desde las comunidades, en tanto que se da un tiempo suficiente donde se posibilite el establecimiento de vínculos con las personas y las comunidades que permitan confianza para una acción de largo aliento.

De igual manera sucede con la llamada "lógica del formato", que busca la evidencia por la evidencia. Parte de una racionalidad instrumental que valora en mayor proporción la eficacia en el logro de objetivos y cumplimiento de metas, recogiendo la evidencia en una lógica formal que es operatoria y calculadora. Este aspecto técnico trae como consecuencia la reducción de la acción humana al trabajo técnico organizado; y de los problemas humanos como problemas técnicos (Contreras, 2006). De hecho, la razón instrumental a la que nos estamos refiriendo sólo tiene capacidad de comprender aquello que abarca su propia lógica, lo demás queda relegado. Entonces temas como la sensibilidad, el vínculo, la confianza, la escucha, la disposición hacia otro, son ignorados muchas veces en esta lógica de atención.

Zygmunt Bauman (1989) nos proporciona un elemento adicional para la reflexión cuando plantea en "Modernidad y Holocausto" la manera como el sistema de la burocracia se implanta como régimen en un entramado social: se instala una lógica ciega de obediencia, de cumplimiento de deberes sin reflexión, donde las "obligaciones morales se pueden concentrar por completo en hacer bien su trabajo, la moralidad se desvanece ante el empeño de ser bueno, eficiente y diligente trabajador y experto" (p. 128). De allí el primado de la estadística, puesto que es un lenguaje que mide resultados, a través de diagramas y gráficos, sin emitir juicios morales, en donde lo único que importa es el éxito o fracaso de la cifra, de lo cuantificable, perdiendo de esta forma la peculiaridad y el propósito de la acción, lo que puede originar un escenario deshumanizante que "comienza ahí, gracias al distanciamiento, (pues) los objetos hacia los que se dirige la operación burocrática se reducen a un conjunto de medidas cuantitativas" (p. 128) al objetivar todas las tareas, las cuales serían parecidas y mecánicas. Sin embargo, el peligro radica en no hacer una lectura más allá de esta mirada cuantificadora, centrada en carencias y problemas; ignorando la funcionalidad y adaptabilidad de algunas respuestas individuales y sociales, o los procesos de resiliencia y resistencia de la gente (Villa, 2014b)

(...) se ha observado, analizado y descrito la tendencia de todas las burocracias a perder de vista el objetivo original y a centrarse en los medios, medios que se convierten en fines... la gente, las cosas y los acontecimientos están programados, se habla de inputs y outputs, de circuitos cerrados de realimentación, de variables, porcentajes, procesos y tal, hasta que finalmente desaparece todo contacto con la situación real. Quedan gráficos, bases de datos e informes impresos (Bauman, 1989, p. 132 - 142).

Según Bauman (1989) y Blanco (2012), la lógica está incapacitada para evitar que se perpetúen problemáticas sociales. Por estar parada en esta cultura burocrática, considera que la sociedad es un objeto a administrar y que desde allí están dadas las soluciones: el modelo de sujeto y de sociedad que se tiene está al servicio de las fuerzas económicas, del poder como instrumento de dominio, "ajena a los valores, agotada en los hechos y en los números, deudora del cálculo y la utilidad material, que capitula ante la realidad existente" (P. 129). Hay que admitirlo, si la 
racionalidad instrumental es la que guía la acción y lo fundamental son procesos técnicos, metas medidas en cifras, llenar formatos, nos encontramos con una acción ciega, centrada en resolver problemas técnicos, e incapaz de responder a las verdaderas vicisitudes, problemas y sufrimientos de la gente (Martín-Baró, 1989; Nusbbaun, 2011); pero además, generadora de desgaste emocional y malestar ético de los profesionales que trabajan, desde sentidos de vida construidos en perspectiva de transformación social, quienes a la larga terminan o bien, "quemados" y saliendo de los proyectos, o bien asumiendo posiciones de distancia emocional, dureza e indolencia, con el fin de poder cumplir objetivos, satisfacer necesidades laborales y económicas y hacer el trabajo que demanda la institucionalidad de la mejor manera posible.

Si a este problema se le agrega el del clientelismo, se puede afirmar que tanto los y las profesionales, como las poblaciones refuerzan una visión del Estado como un ente inoperante, que no responde a las necesidades vitales y reales de la gente, que desperdicia recursos en acciones que no tienen sentido, que no es eficiente para superar los problemas reales de las poblaciones, y que además es clientelista, en donde los funcionarios y sus recursos son tratados y recibidos como intercambio de favores (votos, lealtades, etc), con lo que se obstaculiza la construcción de la confianza necesaria en la relación Estado-ciudadano, fundamental para acompañar todo proceso de recuperación y reparación social (Bello \& Millan, 2005).

\section{Conclusiones.}

De acuerdo con los relatos de los profesionales entrevistados, los proyectos de intervención psicosocial tendrían que contemplar un tiempo prolongado para los procesos y para la contratación de los profesionales; el conocimiento del contexto, la planeación de los proyectos y su capacitación, para responder a las necesidades reales y concretas de la gente.

Paralelo a esto, se resalta la importancia de quienes realizan la selección del personal, puesto que se requieren personas que, al trabajar con personas en situaciones vulnerables, sea éste uno de sus sentidos de vida y una convicción; y no como la posibilidad de tener un buen ingreso económico, o en el cumplimiento de un favor político, en un lugar de indolencia frente a lo que al otro le sucede.

Por otro lado, el tema de trabajar en un contexto en donde aún hay violencia y necesidades básicas insatisfechas, es complejo para pensar una reparación integral y una transformación de la sociedad, puesto que, para ello, también se deben subsanar estas necesidades y carencias que tiene la población, además de superar las barreras en los accesos a diferentes derechos que debe garantizar el Estado (salud, trabajo, educación).

No hay que perder de vista, que la mayoría de las personas víctimas son personas en condiciones de pobreza significativa, y que esta situación muchas veces los lleva a parecer "mendigos" de atenciones (Villa \& Insuasty, 2015), y que ese debe ser uno de los puntos centrales a trabajar con ellos, para lograr una verdadera transformación que promueva el empoderamiento, la autogestión y la reconstrucción de su tejido social. 


\section{Bibliografía.}

AVRE. (2010). Reflexiones desde la práctica de acompañamiento psicosocial y en salud mental: lecciones aprendidas. Bogotá: Arfo Editores e Impresores Ltda.

Bauma, Z. (1989). Modernidad y Holocausto. Madrid: Sequitur.

Bello, M. N., \& Millán, C. (2005). La intervención institucional en contextos culturalmente distintos: Lógicas en tensión y contradicción. En M. N. Bello, E. Martín, C. Millán, B. Pulido, \& R. Rojas, Bojayá, Memoria y Río. Violencia pólitica, Daño y Reparación. Bogotá: Editorial Unibiblos.

Blanco, A. (2012). La exigencia de la praxis, clave teórica de la psicología de la liberación. En D. Montero, \& P. (. Fernández de Larrinoa, Calidad de vida, inclusión social y procesos de intervención (págs. 15-60). Bilbao: Publicaciones de la Universidad de Deusto.

Blanco, A., \& Gaborit, M. (2014). La razón compasiva y la acción como ideología: una nueva mirada a la Psicología de la liberación. En E. Zubieta , Psicología social y Psicología política: procesos teóricos y estudios aplicados (págs. 139-170). Buenos Aires: Eudeba.

Clancy, M., \& Hamber, B. (2008). Trauma, Peacebuilding and development: An overview of key position and critical question. Trauma, Desarrollo y Construcción de Paz, (págs. 9-11). Nueva Delhi, India.

Contreras, F. (2006). Estudio crítico de la razón instrumental totalitaria. Revista científica de información y comunicación, 63-84.

Das, V. (2008). La Antropología del Dolor. En F. Ortega, Veena Das: Sujetos del dolor, agentes de dignidad (págs. 409-436). Bogotá: Pontificia Universidad Javeriana y Universidad Nacional de Colombia.

Fernández Christlieb, P. (2011). Lo psicosocial . En A. Ovejero, \& J. Ramos, Psicología social crítica (págs. 48-57). México: Biblioteca Nueva, Universidad de Querétaro.

Flick, U. (2004). Introducción a la investigación cualitativa. Madrid: Ediciones Morata y Fundación Paideia.

Martín Beristain, C. (1999). La experiencia de cooperantes y ONG. En C. Martín Beristaín, G. Dona, D. Páez, P. Pérez, \& I. Fernández, Reconstruir el tejido social (págs. 137-145). Barcelona: Icaria editorial.

Martín Beristain, C. (2010). Manual sobre perspectiva psicosocial en la investigación de derechos humanos. Bilbao: Cejil, Universidad del País Vasco y Hegoa.

Martín Beristain, C., \& Dona, G. (1997). Enfoque Psicosocial de la ayuda humanitaria. Bilbao: Universidad de Deusto, Instituto de Derechos Humanos. 
Martín-Baró , I. (1983). Acción e Ideología: Psicología social desde Centroamérica I. San Salvador: UCA Editores.

Martín-Baró , I. (1989). Guerra y Salud Mental. San Salvador: UCA Editores.

Martín-Baró, I. (1998). El papel desenmascarador del psicólogo. En I. Martín-Baró, A. Blanco, \& N. Chomsky, Psicología de la liberación (págs. 161-199). Madrid: Trotta Editorial.

Martín-Baró, I. (2006). Hacia una psicología de la liberación. Psicología sin fronteras. Revista electrónica de intervención psicosocial y psicología comunitaria, 1(2), 7-14.

Montenegro, M. (2001). Intervenciones participativas. En Psicología Social Comunitaria. Barcelona: Universidad Autónoma.

Nussbaum, M. (2010). Sin Fines de Lucro: ¿Por qué la democracia necesita de las humanidades? Madrid: Katz.

Sandoval, C. (1996). Investigación cualitativa. Bogotá: ICFES.

Villa Gómez, J. D. (2012). La acción y el enfoque psicosocial de la intervención en contextos sociales: ¿podemos pasar de la moda a la precisión teórica, epistemológica y metodológica? El Ágora USB, 12(2), 349-365. Obtenido de http://revistas.usb.edu.co/index.php/Agora/article/view/208

Villa Gómez, J. D. (2013). Horizontalidad, expresión y saberes compartidos: enfoque psicosocial en procesos de acompañamiento a víctimas de violencia política en Colombia. El Ágora USB, 13(1), 289-327. Obtenido de http://revistas.usb.edu.co/index.php/Agora/article/view/91

Villa Gómez, J. D. (2014a). Recordar para Reconstruir. Medellín: Editorial Bonaventuriana.

Villa Gómez, J. D. (2014b). Un análisis crítico a la intervención psicosocial con víctimas del conflicto armado en Colombia. Bogotá: Ponencia presentada en la IX Cátedra Internacional Martín-Baró, Pontificia Universidad Javeriana.

Villa Gomez, J. D., \& Insuasty Rodríguez, A. (2015). Significados en torno a la reparación, la ayuda humanitaria, la indemnización y la restitución en víctimas del conflicto armado en el municipio de San Carlos. El Agora USB, 15(2), 419-445. Obtenido de http://revistas.usb.edu.co/index.php/Agora/article/view/1623

Villa Gómez, J. D., Arroyave, L., Montoya, Y., \& Muñoz, A. (2015). Aspectos vocacionales y sentidos de vida en profesionales que trabajan en proyectos de atención psicosocial a víctimas de violencia política en Medellín y Antioquia. Revista de Psicología, 7(2), 9-30. 